\title{
A Theoretical Study on the Substrate Deacylation Mechanism of Class C $\beta$-Lactamase
}

\author{
Masayuki Hata, ${ }^{*}$ Yoshikazu Tanaka, Yasuyuki Fujii, Saburo Neya and Tyuji Hoshino
}

\author{
Supporting Information
}

\section{(TS3)}

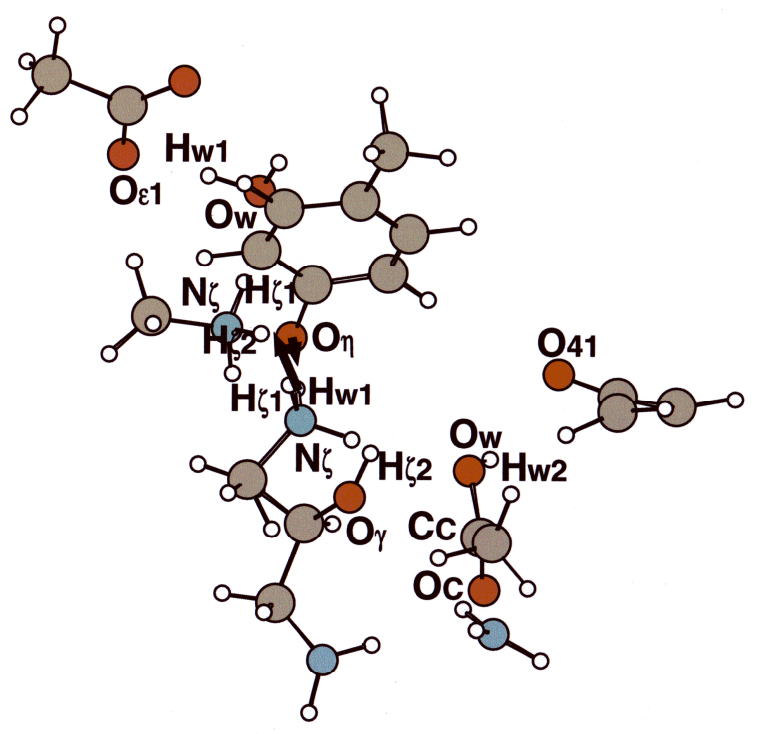

(+ $25.96 \mathrm{kcal} / \mathrm{mol})$
(S3)

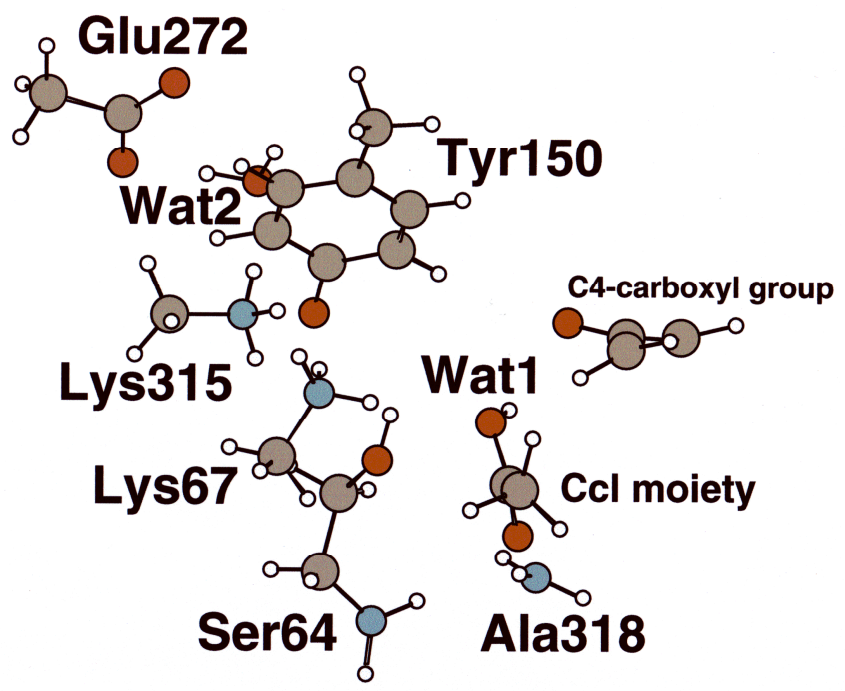

H : O

C: : $\bigcirc$

(+ $24.48 \mathrm{kcal} / \mathrm{mol})$
$\mathrm{N}: \mathrm{O}$

$0: 0$

Figure S1. TS structure (TS3) and product structure (S3) obtained by IRC calculation of the proton transfer from Tyr150 to Lys67. Arrows in (TS3) indicate vibrational modes obtained by normal frequency analysis. Atom names and residue names are shown in (TS3) and (S3), respectively. Numerals in parentheses are relative energies to the potential energy of (S2). 
Table S1. Important inter-atomic distances in the structures of TS3 and S3 obtained by IRC calculation of the proton transfer from Tyr150 to Lys67.

\begin{tabular}{|c|c|c|}
\hline Inter-atomic distance $(\AA)$ & $\mathrm{TS} 3$ & S3 \\
\hline Wat $1 \mathrm{O}_{\mathrm{w}}-\mathrm{CclC}_{\mathrm{c}}$ & 1.33 & 1.32 \\
\hline Wat $1 \mathrm{O}_{\mathrm{w}}-\mathrm{Tyr} 150 \mathrm{O}_{\eta}$ & 4.26 & 4.12 \\
\hline Wat $1 \mathrm{O}_{\mathrm{w}}-\mathrm{CclO}_{41}$ & 2.91 & 2.91 \\
\hline $\mathrm{Wat}_{1} \mathrm{H}_{\mathrm{w} 2}-\mathrm{CclO}_{41}$ & 2.14 & 2.15 \\
\hline Wat1 $\mathrm{H}_{\mathrm{w} 1}-\mathrm{Tyr} 150 \mathrm{O}_{\eta}$ & 1.36 & 1.61 \\
\hline Wat1H ${ }_{w 1}-$ Lys67N & 1.15 & 1.05 \\
\hline Ser64O $_{\gamma}-$ Lys67H $\mathrm{H}_{\zeta 2}$ & 0.95 & 0.95 \\
\hline Ser64O ${ }_{\gamma}-$ Lys67N $_{\zeta}$ & 2.69 & 2.71 \\
\hline $\mathrm{Ser} 4 \mathrm{O}_{\gamma}-\mathrm{CclC}_{\mathrm{c}}$ & 2.62 & 2.63 \\
\hline Tyr150O - -Lys67N & 2.49 & 2.63 \\
\hline Tyr150O ${ }_{\eta}-$ Lys67H $\mathrm{H}_{\zeta 1}$ & 2.84 & 2.91 \\
\hline Tyr $150 O_{\eta}-$ Lys $315 \mathrm{~N}_{\zeta}$ & 2.68 & 2.65 \\
\hline Tyr150O ${ }_{\eta}-$ Lys $315 \mathrm{H}_{\zeta 2}$ & 1.67 & 1.61 \\
\hline Lys $315 \mathrm{~N}_{\zeta}-$ Wat $2 \mathrm{O}_{\mathrm{w}}$ & 2.78 & 2.81 \\
\hline Lys $315 \mathrm{H}_{\zeta 1}-$ Wat $2 \mathrm{O}_{\mathrm{w}}$ & 1.77 & 1.81 \\
\hline Glu2 $272 \mathrm{O}_{\varepsilon 1}-$ Wat $2 \mathrm{O}_{\mathrm{w}}$ & 2.62 & 2.62 \\
\hline Glu $272 \mathrm{O}_{\varepsilon 1}-\mathrm{Wat} 2 \mathrm{H}_{\mathrm{w} 1}$ & 1.66 & 1.66 \\
\hline $\mathrm{CclO}_{\mathrm{c}}-\mathrm{Ser64N}$ & 3.19 & 3.16 \\
\hline $\mathrm{CclO}_{\mathrm{c}}-\mathrm{Ala} 318 \mathrm{~N}$ & 2.78 & 2.79 \\
\hline
\end{tabular}

